\title{
RECENT EXPERIMENTAL RESULTS AND MODELLING OF HIGH-MACH-NUMBER JETS AND THE TRANSITION TO TURBULENCE
}

\author{
P.A. ROSEN ${ }^{1}$, B.H. WILDE ${ }^{2}$, R.J.R. WILLIAMS ${ }^{1}$, J.M. FOSTER ${ }^{1}$, P.A. KEITER ${ }^{2}$, \\ R.F. COKER ${ }^{2}$, T.S. PERRY ${ }^{3}$, M.J. TAYLOR ${ }^{1}$, A.M. KHOKHLOV ${ }^{4}$, R.P. DRAKE ${ }^{5}$, \\ G.R. BENNETT ${ }^{6}$, D.B. SINARS ${ }^{6}$ and R.B. CAMPBELL ${ }^{6}$ \\ ${ }^{1} A W E$, Aldermaston, UK; E-mail: paula.rosen@awe.co.uk \\ ${ }^{2}$ Los Alamos National Laboratory, USA \\ ${ }^{3}$ Lawrence Livermore National Laboratory, USA \\ ${ }^{4}$ University of Chicago, USA \\ ${ }^{5}$ University of Michigan, USA \\ ${ }^{6}$ Sandia National Laboratories, Albuquerque, USA
}

(Received 21 April 2004; accepted 31 May 2005)

\begin{abstract}
In recent years, we have carried out experiments at the University of Rochester's Omega laser in which supersonic, dense-plasma jets are formed by the interaction of strong shocks in a complex target assembly (Foster et al., Phys. Plasmas 9 (2002) 2251). We describe recent, significant extensions to this work, in which we consider scaling of the experiment, the transition to turbulence, and astrophysical analogues. In new work at the Omega laser, we are developing an experiment in which a jet is formed by laser ablation of a titanium foil mounted over a titanium washer with a central, cylindrical hole. Some of the resulting shocked titanium expands, cools, and accelerates through the vacuum region (the hole in the washer) and then enters a cylinder of low-density foam as a jet. We discuss the design of this new experiment and present preliminary experimental data and results of simulations using AWE hydrocodes. In each case, the high Reynolds number of the jet suggests that turbulence should develop, although this behaviour cannot be reliably modelled by present, resolution-limited simulations (because of their low-numerical Reynolds number).
\end{abstract}

Keywords: jets, turbulence, laboratory astrophysics, supernovae

\section{Introduction}

Jet-like features are observed in many different kinds of astrophysical objects such as young stellar objects, active galactic nebulae and planetary nebulae. Recent theories and calculations (Khokhlov et al., 1999) imply that jets might also play a principal role in the explosion of core collapse supernovae. Recent observations of core collapse supernovae provide evidence that the explosion is intrinsically asymmetric. For example, jet-like features have been observed in the remnants of supernovae, such as the radio image of SNR W50 and in X-ray images of the Vela pulsar. Khokhlov has developed an explosion model which assumes that bi-polar, non-relativistic jets form via a magneto-rotational mechanism during a 
core collapse, and this results in the production of a highly asymmetric supernova envelope.

There are many facets of jets which are not yet fully understood, in particular the processes by which the strongly collimated flows are driven. Of particular interest is the question of whether and when these jets become turbulent. This has important implications for the range of jets and the manner in which their material and energy content is deposited, as well as for the entrainment and acceleration of material in their envelopes. Reynolds numbers in supernovae are predicted to be astronomical $\left(\sim 10^{10}\right)$, but, to date, none of the simulations has gone turbulent (Kifonidis et al., 2000). It is suspected that the reason for this is that the numerical Reynolds number in these simulations is too low; i.e. the problem cannot be resolved finely enough to calculate mixing at the required scale lengths over which instability growth and turbulent dissipation occur. Many hydrocodes codes are therefore developing sub-grid-scale mix models to capture these effects.

An experimental platform using the Omega laser (Soures et al., 1996) to generate astrophysically relevant jets is being developed to validate these models, and to better understand the physics of jets. We have built upon our success at studying hydrodynamic jets at early time ( $t<15 \mathrm{~ns}$ ) (Foster et al., 2002), and modified our platform to study the jet at late time. Our earlier experiments used a laser-heated hollow cylindrical gold hohlraum (Kauffman et al., 1994; Lindl, 1995) to radiatively drive the targets. However, axial plasma stagnation within the hohlraum generates additional pressure at the target which perturbs the hydrodynamics of the jet. It is for this reason that our current experiments use direct laser drive.

In addition to our experiments at Omega, we are also developing large field-ofview imaging at the $\mathrm{Z}$ pulsed-power facility (SNLA) using a monochromatic curved crystal imager at $6.151 \mathrm{keV}$ (Sinars et al., 2003). This experimental configuration offers the exciting potential of increased effective spatial resolution, but this work will not be discussed further in this paper, due to limitation of space.

\section{Experiment Design}

A diagram of the target is shown in Figure 1. We use between three and seven beams of the Omega laser to irradiate a $125-\mu \mathrm{m}$ thickness titanium foil in direct contact with a 700- $\mu \mathrm{m}$ thickness titanium washer with a central, 300- $\mu \mathrm{m}$ diameter cylindrical hole. A $2-\mu \mathrm{m}$ thickness layer of solid $\left(1.0 \mathrm{~g} \mathrm{~cm}^{-3}\right)$ plastic (Parylene$\mathrm{N}$ ) is placed on the driven side of the titanium to constrain the expansion of hot titanium coronal plasma (for diagnostic reasons). Laser ablation of the surface launches a shock that penetrates through the titanium, some of which expands, cools, and accelerates through the vacuum region. Lateral shocks emanating from the sides of the cylindrical hole act to collimate the accelerating titanium and it penetrates the 6-mm-length, low-density, resorcinol-formaldehyde (RF), aerogel foam as a high-Mach-number jet. The density of the RF foam is $0.1 \mathrm{~g} \mathrm{~cm}^{-3}$, its 


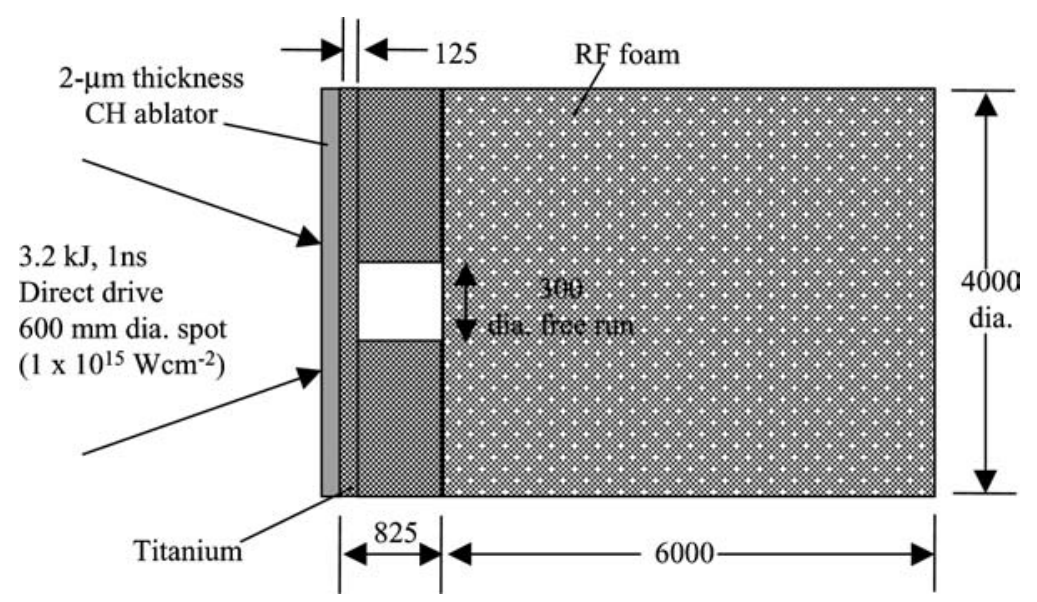

Figure 1. The experimental package for the titanium jet experiment comprises a titanium foil mounted on to a titanium washer. The washer has a central cylindrical hole. Adjacent to the washer is a cylindrical hydrocarbon-foam block. A plastic ablator covers the driven face of the target. Dimensions are in microns.

chemical composition is $\mathrm{C}_{15} \mathrm{H}_{12} \mathrm{O}_{4}$, and it has a cell size of less than $0.1 \mu \mathrm{m}$. The exterior diameter of all components is $4 \mathrm{~mm}$. We use between 1.5 and $3.2 \mathrm{~kJ}$ of laser energy in a 1-ns-length square pulse of $0.35 \mu \mathrm{m}$ wavelength. Super-Gaussian phase plates are used to smooth the incident laser intensity profile; these give a $600-\mu \mathrm{m}$ diameter spot at the $50 \%$ intensity point $(825-\mu \mathrm{m}$ diameter at the $5 \%$ intensity point).

As our images are relatively large (several $\mathrm{mm}$ in size) we diagnose the jet using pinhole-apertured, point-projection X-ray backlighting. (Our earlier experiments studied smaller jets and used wide-area backlighting.) We use vanadium as the backlighter material to provide good contrast with the titanium jet. (The He-like resonance line of vanadium (at $5.205 \mathrm{keV}$ ) lies just above the $\mathrm{K}$-absorption edge of titanium). The vanadium backlighter foil is illuminated with two laser beams ( $\sim 450 \mathrm{~J}$ per beam) in a 1 -ns duration laser pulse $(0.35 \mu \mathrm{m}$ wavelength).

A major challenge for this experiment has been the provision of adequate shielding for this target assembly, and early experimental campaigns were devoted to understanding the source of background radiation.

\section{Simulations}

The experiment is being modelled using the RAGE (Gittings, 1992), LASNEX (Zimmerman and Kruer, 1975) and ALLA (Khokhlov, 1998) hydrocodes, as well as the AWE hydrocodes NYM (Roberts et al., 1980), PETRA (Youngs, 1982, 1984) and TURMOIL3D (Youngs, 1994). Due to limitation of space, only the AWE calculations will be described in detail in this paper. 
NYM is a 2D Lagrangian hydrocode which includes laser ray trace and laser energy deposition packages, and non-local-thermodynamic equilibrium (NLTE) physics. The laser light is input as a $600-\mu \mathrm{m}$ diameter spot on the axis of the calculation. Laser energy absorption is modelled as inverse bremsstrahlung in the coronal plasma, with a 30\% deposition of energy at the critical surface. Radiation transport is treated by Monte Carlo photonics (Fleck and Cummings, 1971). NLTE physics in the laser absorption region is treated by the ZEUS package, which provides in-line time-dependent NLTE modelling by solutions of rate equations for average ion populations in the screened hydrogenic approximation. This NLTE treatment is applied in selected cells of the simulation, including and bordering the region of laser transport and energy deposition; elsewhere, tabular LTE opacities are used, calculated off-line using the IMP (Rose, 1992) opacity code. Equation of state is also input in tabular form, using the SESAME (Lyon and Johnson, 1992) database.

The NYM calculation proceeds until approximately $2 \mathrm{~ns}$ after the beginning of the laser pulse. After this time, the calculational timestep becomes impracticably small, and we transfer the simulation to the AWE 2D Eulerian hydrocode PETRA. In this phase of the calculation, no further energy is input from an external source and the code tracks the further evolution of the jet. Radiation transfer is treated by single-group (grey) diffusion. Ion, electron and radiation temperatures are assumed equal (1-T model). Square, $5 \mu \mathrm{m}$ zones are used in the central part of the Eulerian problem (up to a radius of $500 \mu \mathrm{m}$ ), then the size of the radial zones increases geometrically, with an increase in size of $10 \%$ per cell.

Finally, we post-process the simulation to produce a synthetic radiograph for comparison with the experimental data.

Figure 2 shows plots of log density, titanium volume fraction and a simulated radiograph at $t=300 \mathrm{~ns}$ from a NYM-PETRA simulation. Coarseness of the
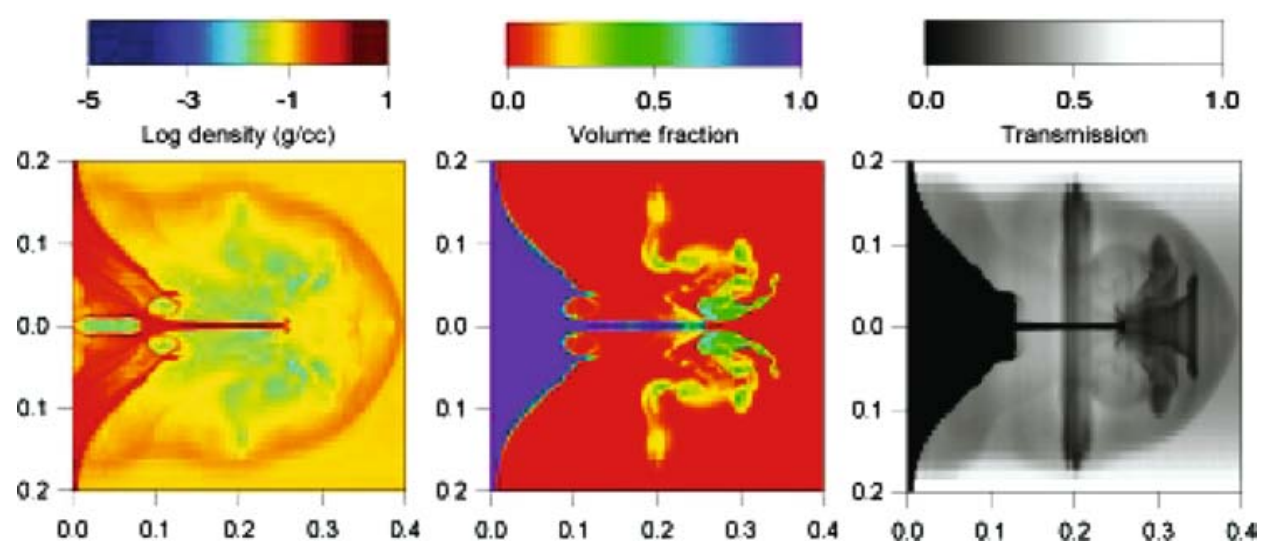

Figure 2. Log density (left), titanium volume fraction and simulated radiograph (right) from a NYMPETRA calculation at $t=300 \mathrm{~ns}$. Dimensions are in centimetres. 

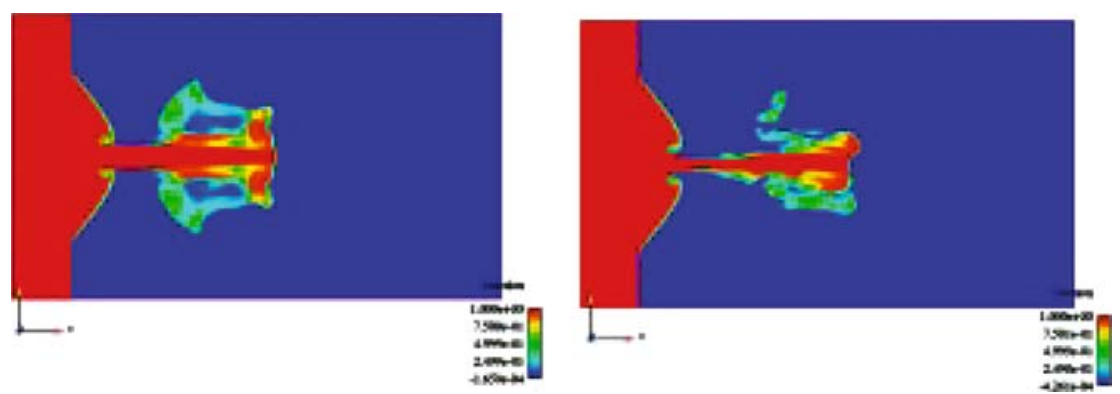

Figure 3. Titanium mass fraction from TURMOIL3D simulations. The left-hand image is from an unperturbed simulation and the right-hand image is from a simulation in which a $20 \mu \mathrm{m}$ random roughness was introduced on the inner surface of the hole in the titanium washer.

calculational mesh is evident at larger radii. The jet exhibits large-scale structures but does not have sufficient resolution to look "turbulent."

These experiments are also being modelled using a three-dimensional Eulerian code, TURMOIL3D, which treats the flow of polytropic gases using perfect-gas equations of state. The code uses a finite-difference staggered-grid Lagrangianremap algorithm, extended from the two-dimensional method used in PETRA (but without interface reconstruction). The remap phase uses the third-order monotonic method of Van Leer (1977) to maintain sharp contact discontinuities without producing negative densities. The code includes the facility to advect the mass fractions of two distinct species, including the variation in the adiabatic index as a function of the composition. Figure 3 shows plots of titanium mass fraction from two TURMOIL3D calculations: an unperturbed simulation and one where $20-\mu \mathrm{m}$ random perturbations have been introduced on the inner surface of the hole in the titanium washer.

\section{Scaling the Jet}

The morphology of the jets created in our experiment shows many points of similarity with numerical models of astrophysical supersonic jets, and the question arises of the extent to which the experiment can be scaled to systems of vastly different physical size. Such scaling has been studied in detail by Ryutov et al. $(1999,2001)$ and Ryutov and Remington (2002) who show that if dissipative processes are negligible, then scaling transformations exist that enable the hydrodynamic evolution of the laboratory system to be mapped onto that of another system at different physical size, providing that certain dimensionless scaling parameters are common to both systems.

From the NYM-PETRA simulation shown in Figure 2, we examine the collimated part of the jet and the region of Kelvin-Helmholtz roll-up. Table I includes values for typical conditions within these parts of the problem together with the 


\section{TABLE I}

Representative physical conditions, and corresponding dimensionless scaling numbers, in the fullydeveloped jet at $t=300 \mathrm{~ns}$, and at the He-H interface of SN1987a at $t=2000 \mathrm{~s}$

\begin{tabular}{lllll}
\hline Quantity & Symbol & $\begin{array}{l}\text { Value in collimated } \\
\text { stem of jet }\end{array}$ & $\begin{array}{l}\text { Value in roll-up } \\
\text { at head of jet }\end{array}$ & $\begin{array}{l}\text { Value in } \\
\text { SN1987a }\end{array}$ \\
\hline Temperature & $T$ & 0.3 & 3 & $900 \mathrm{eV}$ \\
Density & $\rho$ & 4 & 0.1 & $0.0075 \mathrm{~g} \mathrm{~cm}^{-3}$ \\
Pressure & $P$ & $1 \times 10^{11}$ & $1.5 \times 10^{10}$ & $3.5 \times 10^{13} \mathrm{dyn} \mathrm{cm}^{-2}$ \\
Mean ionisation & $Z$ & 3 & 1.4 & 2.0 \\
Fluid velocity & $u$ & $1 \times 10^{6}$ & $1 \times 10^{6}$ & $2 \times 10^{7} \mathrm{~cm} \mathrm{~s}^{-1}$ \\
Length scale & $r$ & $4 \times 10^{-3}$ & $4 \times 10^{-3}$ & $9 \times 10^{10} \mathrm{~cm}^{-3}$ \\
Sound speed & $c_{\mathrm{s}}=\sqrt{\frac{(Z+1) k T}{m_{i}}}$ & $1.6 \times 10^{5}$ & $3.8 \times 10^{5}$ & $2.6 \times 10^{7} \mathrm{~cm} \mathrm{~s}^{-1}$ \\
Internal Mach number & $M=u / c_{s}$ & 6.2 & 2.6 & 0.8 \\
Kinematic viscosity & $v$ & $1.7 \times 10^{-8}$ & $4.5 \times 10^{-3}$ & $7.0 \times 10^{7} \mathrm{~cm}^{2} \mathrm{~s}^{-1}$ \\
Reynolds number & $R e=u r / v$ & $2.4 \times 10^{11}$ & $1 \times 10^{6}$ & $2.6 \times 10^{10}$ \\
Euler number & $E u=u \sqrt{\frac{\rho}{P}}$ & 6.3 & 2.6 & 0.29 \\
\hline
\end{tabular}

${ }^{a}$ Spitzer-Braginskii ion viscosity (Spitzer, 1962).

Reynolds, (internal) Mach and Euler numbers. For purposes of comparison, Table I also includes corresponding data at the (Rayleigh-Taylor unstable) He-H interface of SN1987a at 2000 s (Ryutov et al., 1999).

These values of Re are above the threshold for the transition to turbulence (Dimotakis, 2000), and thus we would expect to see turbulence develop in this experiment, after sufficient elapse of time.
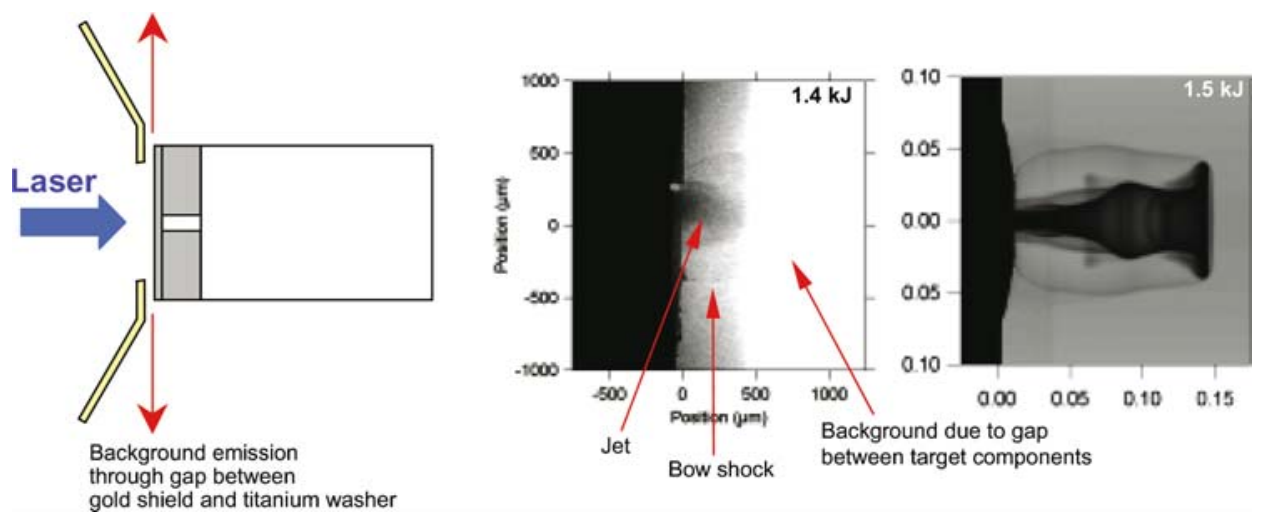

Figure 4. Preliminary experimental data from the titanium jet experiment at $t=100 \mathrm{~ns}$. Parts of the jet and bow shock are visible. The over-exposed part of the image is due to leakage of radiation through a gap between the washer and shield. A simulated radiograph from a NYM-PETRA calculation is qualitatively similar to the data. 


\section{Comparison of Preliminary Experimental Results with Simulation}

Figure 4 shows preliminary experimental data at $t=100 \mathrm{~ns}$ with a simulated radiograph from NYM-PETRA. The experimental data clearly show evidence of a jet and bow shock, but the data are compromised by background X-ray emission which has leaked through a gap between the titanium washer and a gold target shield used as part of the experimental assembly. Several modifications to the target and backlighter configuration have been identified which should eliminate the problem of background radiation, and these will be implemented in future experiments.

\section{Summary}

We are developing a platform to study astrophysically relevant jets at late time (up to several hundred ns). Preliminary experimental results are encouraging and we are optimistic that the next experiment (March 2004) will return high-quality data.

Many different hydrocodes employing different calculation schemes are being used to calculate this experiment. None of the simulations to date shows the transition to turbulence, and it is believed that this is because the numerical Reynolds number is too low (i.e. the finest resolution achievable on today's computers is not high enough to resolve turbulent length scales) or perhaps that there is insufficient time for the transition to turbulence at the numerical Reynolds numbers obtained (Zhou et al., 2003a,b).

These experiments will provide data to test subgrid-scale dynamic mix models in our codes.

\section{Acknowledgements}

The authors would like to thank Jim Knauer (LLE) for helpful discussions on point projection imaging, Chuck Source (LLNL) for diagnostic support, the Omega laser operations team and target fabrication groups at AWE and LLNL. Finally, we are indebted to Bruce Remington and Adam Frank for their motivation, encouragement and support in the pursuit of laboratory analogues of astrophysical jets.

\section{References}

Dimotakis, P.E.: 2000, J. Fluid Mech. 409, 69.

Fleck, J.A. and Cummings, J.D.: 1971, J. Comput. Phys. 8, 313.

Foster, J.M. Wilde, B.H., Rosen, P.A., Perry, T.S., Fell, M., Edwards, M.J., Lasinski, B.F., Turner, R.E. and Gittings, M.L.: 2002, Phys. Plasmas 9, 2251. 
Gittings, M.L.: 1992, Numerical Methods Symposium, April 28-30. Copies may be ordered from the Defence Nuclear Agency, 56801 Telegraph Road, Alexandria, VA 22310-3398.

Khokhlov, A.M.: 1998, J. Comput. Phys. 143, 519.

Khokhlov, A.M., Hoflich, P.A., Oran, E.S., Wheeler, J.C., Wang, L. and Chtchelkanova, A.Yu.: 1999, ApJ 524, 107.

Kauffman, R.L., Suter, L.J., Darrow, C.B., Kilkenny, J.D., Kornblum, H.N., Montgomery, D.S., Phillion, D.W., Rosen, M.D., Theissen, A.R., Wallace, R.J. and Ze, F.: 1994, Phys. Rev. Lett. 73, 2320.

Kifonidis, K., Plewa, T., Janka, H.-T.H. and Muller, E.: 2000, ApJ 531, 123.

Lindl, J.: 1995, Phys. Plasmas 2, 3933.

Lyon, S.P. and Johnson, J.D.: 1992, "Sesame: The Los Alamos National Laboratory Equation of State Database", Los Alamos National Laboratory, Los Alamos, NM, LA-UR-92-3407.

Roberts, P.D., Rose, S.J., Thompson, P.C. and Wright, R.J.: 1980, J. Phys. D 13, 1957.

Rose, S.J.: 1992, J. Phys. B 25, 1667.

Ryutov, D.D., Drake, R.P., Kane, J., Liang, E., Remington, B.A. and Wood-Vesey, W.M.: 1999, ApJ 518,821 .

Ryutov, D.D., Remington, B.A., Robey, H.F. and Drake, R.P.: 2001, Phys. Plasmas 8, 1804.

Ryutov, D.D. and Remington, B.A.: 2002, Plasma Phys. Controlled Fusion 44, B407.

Sinars, D.B., Cuneo, M.E., Bennett, G.R., Wenger, et al.: 2003, Rev. Sci. Instrum. 74, 2202.

Sinars, D.B., Cuneo, M.E. Bennett, G.R., Wenger, Cuneo, M.E. and Porter, J.L.: 2003, Appl. Opt. 42, 4059.

Soures, J., McCrory, R.L. Verdon, C.P., et al.: 1996, Phys. Plasmas 3, 2108.

Spitzer, L.: 1962, The Physics of Fully Ionised Gases, 2nd edn., Wiley, New York, pp. 143.

Van Leer, B.: 1977, J. Comput. Phys. 23, 276.

Youngs, D.L.: 1982, Time-dependent multi-material flow with large fluid distortion, in: K. W. Morton and M. J. Baines (eds.), Numerical Methods for Fluid Mechanics.

Youngs, D. L.: 1984, Physica 12D, 32.

Youngs, D.L.: 1994, Laser and particle beams, 12, 725.

Zhou, Y., Remington, B.A., Robey, H.F., et al.: 2003a, Phys. Plasmas 10, 1883.

Zhou, Y., Robey, H.F. and Buckingham, A.C.: 2003b, Phys. Rev. E 67, 056305-1-11.

Zimmerman, G. and Kruer, W.: 1975, Comments Plasma Phys. Controlled Fusion 11, 51. 